\title{
A Hopf bifurcation in the Kuramoto-Daido model
}

\author{
Institute of Mathematics for Industry, Kyushu University / JST PRESTO, \\ Fukuoka, 819-0395, Japan \\ Hayato CHIBA 1
}

Oct 11,2016

\begin{abstract}
A Hopf bifurcation in the Kuramoto-Daido model is investigated based on the generalized spectral theory and the center manifold reduction for a certain class of frequency distributions. The dynamical system of the order parameter on a four-dimensional center manifold is derived. It is shown that the dynamical system undergoes a Hopf bifurcation as the coupling strength increases, which proves the existence of a periodic two-cluster state of oscillators.
\end{abstract}

\section{Introduction}

Collective synchronization phenomena are observed in a variety of areas such as chemical reactions, engineering circuits and biological populations [10]. In order to investigate such phenomena, a system of globally coupled phase oscillators called the Kuramoto-Daido model [6]

$$
\frac{d \theta_{i}}{d t}=\omega_{i}+\frac{K}{N} \sum_{j=1}^{N} f\left(\theta_{j}-\theta_{i}\right), i=1, \cdots, N,
$$

is often used, where $\theta_{i}=\theta_{i}(t) \in[0,2 \pi)$ is a dependent variable which denotes the phase of an $i$-th oscillator on a circle, $\omega_{i} \in \mathbb{R}$ denotes its natural frequency drawn from some distribution function $g(\omega), K>0$ is a coupling strength, and where $f(\theta)$ is a $2 \pi$-periodic function. The complex order parameter defined by

$$
r e^{i \psi}:=\frac{1}{N} \sum_{j=1}^{N} e^{i \theta_{j}(t)}, \quad i=\sqrt{-1}
$$




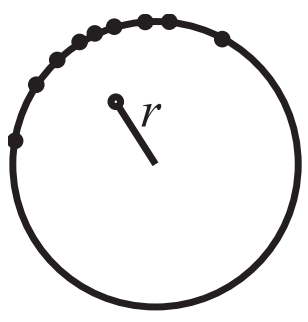

synchronization

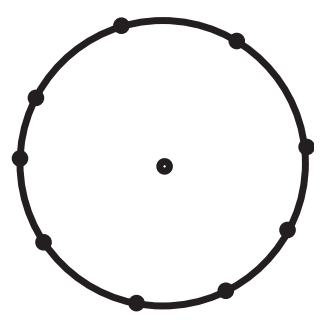

de-synchronization

Figure 1: Collective behavior of oscillators.

is used to measure the amount of collective behavior in the system; if $r$ is nearly equal to zero, oscillators are uniformly distributed (called the incoherent state), while if $r>0$, the synchronization occurs, see Fig. 1,

In this paper, the continuous limit (thermodynamics limit) of the following model

$$
\frac{d \theta_{i}}{d t}=\omega_{i}+\frac{K}{N} \sum_{j=1}^{N}\left(\sin \left(\theta_{j}-\theta_{i}\right)+h \cdot \sin 2\left(\theta_{j}-\theta_{i}\right)\right),
$$

will be considered, where $h$ is a parameter which controls the strength of the second harmonic. For the continuous limit of the system, a Hopf bifurcation from the incoherent state to the two-cluster periodic state will be investigated based on the generalized spectral theory.

It is known that when the frequency distribution $g(\omega)$ is an even and unimodal function, the transition from the incoherent state to the partially synchronized state occurs at the critical coupling strength $K=K_{c}=2 /(\pi g(0))$. In Chiba [1, 2], this result is proved based on the generalized spectral theory [3] under the assumption that $g(\omega)$ has an analytic continuation near the real axis. With the aid of the generalized spectral theory, it is proved that the order parameter is locally governed by the dynamical system on a center manifold as

$$
\frac{d r}{d t}=\text { const. }\left(K-K_{c}+\frac{\pi g^{\prime \prime}(0) K_{c}^{4}}{16} r^{2}\right) r+O\left(r^{4}\right)
$$

for $h=0$, and

$$
\frac{d r}{d t}=\text { const. }\left(K-K_{c}-\frac{K_{c}^{2} C h}{1-h} r\right) r+O\left(r^{3}\right),
$$

\footnotetext{
${ }^{1}$ chiba@imi.kyushu-u.ac.jp
} 
(a)

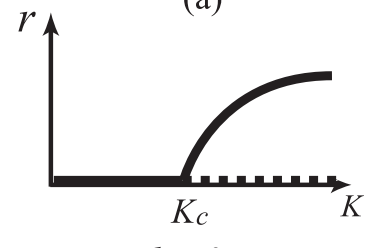

$h=0$

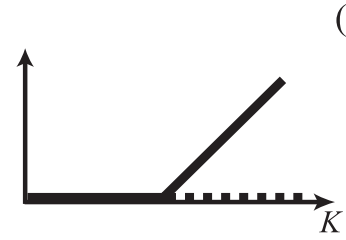

$h<0$ (b)

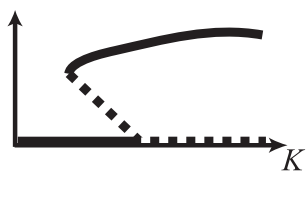

Figure 2: Bifurcation diagrams of the order parameter for (a) $f(\theta)=\sin \theta$ and (b) $f(\theta)=\sin \theta+h \sin 2 \theta$. The solid lines denote stable solutions, and the dotted lines denote unstable solutions.

for $h \neq 0$, where $C$ is a certain negative constant. As a result, a bifurcation diagram of $r$ is given as Fig,2, When $h=0$, the synchronous state emerges through a pitchfork bifurcation, though when $h \neq 0$, it is a transcritical bifurcation.

The purpose in this paper is to investigate a Hopf bifurcation of the system (1.3) under certain assumptions for the distribution function $g(\omega)$. In particular, the dynamics of the order parameter on a center manifold will be derived. For this purpose, we need five assumptions (A1) to (A5) given after Section 3. Here, we give a rough explanation of these assumptions.

(A1) We assume that $h<1$ so that $\sin \theta$ is a dominant term in the coupling function.

(A2) We assume that the distribution $g(\omega)$ of natural frequencies is an analytic function near the real axis. This is the essential assumption to apply the generalized spectral theory.

(A3) We will show that at a bifurcation value $K=K_{c}$, a pair of generalized eigenvalues of a certain linear operator obtained by the linearization of the system locates at the points $\pm i y_{c}$ on the imaginary axis. We assume that such a pair is unique and they are simple eigenvalues.

(A4) We assume that as $K$ increases, the pair of generalized eigenvalues transversally gets across the imaginary axis at the point $\pm i y_{c}$ from the left to the right.

(A5) Assume that $g(\omega)$ is an even function.

It seems that (A3) and (A4) are satisfied for a wide class of even and bimodal distributions $g(\omega)$ as long as the distance of two peaks are sufficiently far apart, though we do not assume explicitly that $g(\omega)$ is bimodal. The main results in the present paper are stated as follows;

Theorem 1.1 (Instability of the incoherent state).

Suppose (A1) and $g(\omega)$ is continuous. There exists a number $\varepsilon>0$ such that 
when $K_{c}<K<K_{c}+\varepsilon$, the incoherent state is linearly unstable, where

$$
K_{c}=\frac{2}{\pi g\left(y_{c}\right)}
$$

and $y_{c}$ is a certain real number, see (A3) above.

Theorem 1.2 (Local stability of the incoherent state).

Suppose (A1) and (A2). When $0<K<K_{c}$, the incoherent state is linearly asymptotically stable in the weak sense (see Section 4 for the weak stability).

\section{Theorem 1.3 (Bifurcation).}

Suppose (A1) to (A5). There exists a positive constant $\varepsilon_{0}$ such that if $K_{c}-$ $\varepsilon_{0}<K<K_{c}+\varepsilon_{0}$ and if an initial condition is closed to the incoherent state, the dynamics of the order parameter is locally governed by a certain four dimensional dynamical system on the center manifold given in Section 6. At $K=K_{c}$ the system undergoes a Hopf bifurcation and when $K_{c}<$ $K<K_{c}+\varepsilon_{0}, h \leq 0$ and $\operatorname{Re}\left(p_{2}\right)<0$ (see below), the system has a family of asymptotically stable periodic orbits.

(i) Suppose $h=0$. On the family of stable periodic orbits, the complex order parameter $\eta_{1}$ defined in Section 2 is given by

$$
\eta_{1}(t)=2 \sqrt{\frac{-\operatorname{Re}\left(p_{1}\right)}{\operatorname{Re}\left(p_{2}\right)}} \sqrt{K-K_{c}} e^{i \beta} \cos \left(y_{c} t+O\left(K-K_{c}\right)\right)+O\left(K-K_{c}\right),
$$

where $p_{1}$ and $p_{2}$ are certain complex constants explicitly given in Section 6, and $\beta \in \mathbb{R}$ is an arbitrary constant specified by an initial condition. The assumption (A4) implies $\operatorname{Re}\left(p_{1}\right)>0$.

(ii) Suppose $h<0$. On the family of stable periodic orbits, the complex order parameter $\eta_{1}$ is given by

$\eta_{1}(t)=-2 \frac{1-h}{h K_{c}} \operatorname{Re}\left(p_{1}\right)\left(K-K_{c}\right) e^{i \beta} \cos \left(y_{c} t+O\left(K-K_{c}\right)\right)+O\left(\left(K-K_{c}\right)^{2}\right)$,

where $p_{1}$ is the same constant as (i).

The constants $p_{1}$ and $p_{2}$ are determined only by the frequency distribution $g(\omega)$. The condition $\operatorname{Re}\left(p_{2}\right)<0$ seems to be satisfied for most even and bimodal distributions. If $\operatorname{Re}\left(p_{2}\right)>0$, a family of unstable periodic orbits exists when $K_{c}-\varepsilon_{0}<K<K_{c}$; that is, a bifurcation occurs in the subcritical regime, while the expression for $\eta_{1}$ is the same as above. Similarly, if $0<$ $h<1$, a bifurcation is subcritical and a family of unstable periodic orbits exists for $K_{c}-\varepsilon_{0}<K<K_{c}$. 
In Martens et. al 8], the following bimodal frequency distribution defined as the sum of two Lorentzian distribution

$$
g(\omega)=\frac{1}{2 \pi}\left(\frac{1}{\left(\omega-\omega_{0}\right)^{2}+1}+\frac{1}{\left(\omega+\omega_{0}\right)^{2}+1}\right)
$$

is considered. They revealed the dynamics of the order parameter in detail by using the Ott-Antonsen ansatz [9], though it is applicable only when $h=0$.

For this bimodal distribution, we can verify that $K_{c}=4, y_{c}=\sqrt{\omega_{0}^{2}-1}$, $\operatorname{Re}\left(p_{1}\right)=1 / 4$ and $\operatorname{Re}\left(p_{2}\right)=-4$. Hence, there exists a family of stable periodic solutions for both of $h=0$ and $h<0$. See also Example 3.5 and Example 5.3.

\section{The continuous model}

For the finite dimensional Kuramoto-Daido model (1.1), the $l$-th order parameter is defined by

$$
\hat{\eta}_{l}(t):=\frac{1}{N} \sum_{j=1}^{N} e^{i l \theta_{j}(t)}
$$

By using it, Eq.(1.1) is rewritten as

$$
\frac{d \theta_{j}}{d t}=\omega_{j}+K \sum_{l=-\infty}^{\infty} f_{l} \hat{\eta}_{l}(t) e^{-i l \theta_{j}}, \quad f(\theta):=\sum_{l=-\infty}^{\infty} f_{l} e^{i l \theta} .
$$

The continuous limit of this model is an evolution equation of a density

$$
\left\{\begin{array}{l}
\frac{\partial \rho_{t}}{\partial t}+\frac{\partial}{\partial \theta}\left(\rho_{t} v\right)=0, \quad \rho_{t}=\rho_{t}(\theta, \omega), \\
v:=\omega+K \sum_{l=-\infty}^{\infty} f_{l} \eta_{l}(t) e^{-i l \theta} \\
\eta_{l}(t):=\int_{\mathbb{R}} \int_{0}^{2 \pi} e^{i l \theta} \rho_{t}(\theta, \omega) g(\omega) d \theta d \omega .
\end{array}\right.
$$

Here, $g(\omega)$ is a given probability density function for natural frequencies, and the unknown function $\rho_{t}=\rho_{t}(\theta, \omega)$ is a probability measure on $[0,2 \pi)$ parameterized by $t, \omega \in \mathbb{R} . \eta_{l}(t)$ is a continuous analog of $\hat{\eta}_{l}(t)$ in (2.1). In particular, $\eta_{1}(t)$ is a continuous version of Kuramoto's order parameter (1.2). The trivial solution $\rho_{t}=1 /(2 \pi)$ of the system is a uniform distribution on the circle, which is called the incoherent state (de-synchronous state). Our 
purpose is to investigate the stability and bifurcation of the incoherent state and the order parameter $\eta_{1}$.

Define the Fourier coefficients

$$
Z_{j}(t, \omega):=\int_{0}^{2 \pi} e^{i j \theta} \rho_{t}(\theta, \omega) d \theta .
$$

Then, the continuous model is rewritten as a system of evolution equations of $Z_{j}$

$$
\frac{d Z_{j}}{d t}=i j \omega Z_{j}+i j K f_{j} \eta_{j}+i j K \sum_{l \neq j} f_{l} \eta_{l} Z_{j-l} .
$$

The trivial solution $Z_{j} \equiv 0(j= \pm 1, \pm 2, \cdots)$ corresponds to the incoherent state $\left(Z_{0} \equiv 1\right.$ because of the normalization $\left.\int_{0}^{2 \pi} \rho_{t}(\theta, \omega) d \theta=1\right)$. In what follows, we consider only the equations for $Z_{1}, Z_{2}, \cdots$ because $Z_{-j}$ is the complex conjugate of $Z_{j}$.

\section{The transition point formula and linear in- stability}

To investigate the stability of the incoherent state, we consider the linearized system. Let $L^{2}(\mathbb{R}, g(\omega) d \omega)$ be the weighted Lebesgue space with the inner product

$$
(\phi, \psi)=\int_{\mathbb{R}} \phi(\omega) \overline{\psi(\omega)} g(\omega) d \omega
$$

We define a one-dimensional integral operator $\mathcal{P}$ on $L^{2}(\mathbb{R}, g(\omega) d \omega)$ by

$$
(\mathcal{P} \phi)(\omega)=\int_{\mathbb{R}} \phi(\omega) g(\omega) d \omega=\left(\phi, P_{0}\right) \cdot P_{0}(\omega),
$$

where $P_{0}(\omega) \equiv 1 \in L^{2}(\mathbb{R}, g(\omega) d \omega)$ is a constant function. Then, the order parameters are written by

$$
\eta_{j}(t)=\int_{\mathbb{R}} Z_{j}(t, \omega) g(\omega) d \omega=\left(Z_{j}, P_{0}\right) \cdot P_{0}(\omega)=\mathcal{P} Z_{j} .
$$

Hence, Eq.(2.3) is expressed as

$$
\frac{d Z_{j}}{d t}=\left(i j \omega+i j K f_{j} \mathcal{P}\right) Z_{j}+i j K \sum_{l \neq j} f_{l}\left(\mathcal{P} Z_{l}\right) Z_{j-l}
$$


The linearized system around the incoherent state is given by

$$
\frac{d Z_{j}}{d t}=T_{j} Z_{j}:=\left(i j \omega+i j K f_{j} \mathcal{P}\right) Z_{j}, \quad j=1,2, \cdots
$$

where $T_{j}=i j \omega+i j K f_{j} \mathcal{P}$ is a linear operator on $L^{2}(\mathbb{R}, g(\omega) d \omega)$. Let us consider the spectra of $T_{j}$. The multiplication operator $\phi(\omega) \mapsto \omega \phi(\omega)$ on $L^{2}(\mathbb{R}, g(\omega) d \omega)$ is self-adjoint. The spectrum of it consists only of the continuous spectrum given by $\sigma_{c}(\omega)=\operatorname{supp}(g)$ (the support of $g$ ). Therefore, the spectrum of the multiplication by $i j \omega$ lies on the imaginary axis; $\sigma_{c}(i j \omega)=i j \cdot \operatorname{supp}(g)$ (later we will suppose that $g$ is analytic, so that $\sigma_{c}(i j \omega)$ is the whole imaginary axis). Since $\mathcal{P}$ is compact, it follows from the perturbation theory of linear operators [7] that the continuous spectrum of $T_{j}$ is given by $\sigma_{c}\left(T_{j}\right)=i j \cdot \operatorname{supp}(g)$, and the residual spectrum of $T_{j}$ is empty.

When $f_{j} \neq 0$, eigenvalues $\lambda$ of $T_{j}$ are given as roots of the equation

$$
\int_{\mathbb{R}} \frac{1}{\lambda-i j \omega} g(\omega) d \omega=\frac{1}{i j K f_{j}} .
$$

Indeed, the equation $\left(\lambda-T_{j}\right) v=0$ provides

$$
v+i j K f_{j}\left(v, P_{0}\right)(\lambda-i j \omega)^{-1} P_{0}=0 .
$$

Taking the inner product with $P_{0}$, we obtain Eq.(3.5). If $\lambda$ is an eigenvalue of $T_{j}$, the above equality shows that

$$
v_{\lambda}(\omega)=\frac{1}{\lambda-i j \omega}
$$

is the associated eigenfunction. This is not in $L^{2}(\mathbb{R}, g(\omega) d \omega)$ when $\lambda$ is a purely imaginary number. Thus, there are no eigenvalues on the imaginary axis. Putting $\lambda=x+i y$ in Eq.(3.5) provides

$$
\left\{\begin{aligned}
\int_{\mathbb{R}} \frac{x}{x^{2}+(y-j \omega)^{2}} g(\omega) d \omega & =\frac{-\operatorname{Im}\left(f_{j}\right)}{j K\left|f_{j}\right|^{2}}, \\
\int_{\mathbb{R}} \frac{y-j \omega}{x^{2}+(y-j \omega)^{2}} g(\omega) d \omega & =\frac{\operatorname{Re}\left(f_{j}\right)}{j K\left|f_{j}\right|^{2}},
\end{aligned}\right.
$$

which determines eigenvalues of $T_{j}$. In what follows, we restrict our problem to the model (1.3), for which the coupling function is given by $f(\theta)=\sin \theta+$ $h \sin 2 \theta$. In this case, we have $f_{1}=1 /(2 i), f_{2}=h /(2 i)$ and $f_{j}=0$ for $j \neq 1,2$. The spectrum of the operator $T_{j}$ for $j \neq 1,2$ consists only of the continuous spectrum on the imaginary axis. $T_{1}$ and $T_{2}$ also have the continuous spectra 
on the imaginary axis. Further, they have eigenvalues determined by the equations

$$
\left\{\begin{array}{c}
\int_{\mathbb{R}} \frac{x}{x^{2}+(y-\omega)^{2}} g(\omega) d \omega=\frac{2}{K} \\
\int_{\mathbb{R}} \frac{y-\omega}{x^{2}+(y-\omega)^{2}} g(\omega) d \omega=0
\end{array}\right.
$$

and

$$
\left\{\begin{array}{c}
\int_{\mathbb{R}} \frac{x}{x^{2}+(y-2 \omega)^{2}} g(\omega) d \omega=\frac{h}{K}, \\
\int_{\mathbb{R}} \frac{y-2 \omega}{x^{2}+(y-2 \omega)^{2}} g(\omega) d \omega=0
\end{array}\right.
$$

respectively. Eq.(3.5) for $j=1$ is given by

$$
D(\lambda):=\int_{\mathbb{R}} \frac{1}{\lambda-i \omega} g(\omega) d \omega=\frac{2}{K} .
$$

The next lemma follows from formulae of the Poisson integral and the Hilbert transform.

Lemma 3.1. Suppose $g(\omega)$ is continuous. Then, the equality

$$
\begin{aligned}
\lim _{\lambda \rightarrow+0+i y} D^{(n)}(\lambda) & =(-1)^{n} n ! \cdot \lim _{\lambda \rightarrow+0+i y} \int_{\mathbb{R}} \frac{1}{(\lambda-i \omega)^{n+1}} g(\omega) d \omega \\
& =\frac{1}{i^{n}} \cdot \lim _{\lambda \rightarrow+0+i y} \int_{\mathbb{R}} \frac{1}{\lambda-i \omega} g^{(n)}(\omega) d \omega \\
& =\frac{1}{i^{n}}\left(\pi g^{(n)}(y)-i \pi H\left[g^{(n)}\right](y)\right)
\end{aligned}
$$

holds for $n=0,1,2, \cdots$, where $\lambda \rightarrow+0+i y$ implies the limit to the point $i y \in i \mathbb{R}$ from the right half plane and $H[g]$ denotes the Hilbert transform defined by

$$
\begin{aligned}
H[g](y) & =\frac{-1}{\pi} \mathrm{p} \cdot \mathrm{v} \cdot \int_{\mathbb{R}} \frac{1}{\omega} g(\omega+y) d \omega \\
& =\frac{-1}{\pi} \lim _{\varepsilon \rightarrow+0} \int_{\varepsilon}^{\infty} \frac{1}{\omega}(g(y+\omega)-g(y-\omega)) d \omega .
\end{aligned}
$$

Lemma 3.2. Suppose $K>0$. Then,

(i) If an eigenvalue $\lambda$ of $T_{1}$ exists, it satisfies $\operatorname{Re}(\lambda)>0$.

(ii) If $K>0$ is sufficiently large, there exists at least one eigenvalue $\lambda$ near infinity on the right half plane.

(iii) If $K>0$ is sufficiently small, there are no eigenvalues of $T_{1}$. 
See [2, 4] for the proof.

Eq.(3.7) combined with Lemma 3.1 yields

$$
\left\{\begin{array}{l}
\lim _{x \rightarrow+0} \int_{\mathbb{R}} \frac{x}{x^{2}+(y-\omega)^{2}} g(\omega) d \omega=\pi g(y)=\frac{2}{K}, \\
\lim _{x \rightarrow+0} \int_{\mathbb{R}} \frac{y-\omega}{x^{2}+(y-\omega)^{2}} g(\omega) d \omega=\pi H[g](y)=0 .
\end{array}\right.
$$

Let $y_{1}, y_{2}, \cdots$ be roots of the equation $H[g](y)=0$, and put $K_{j}=2 /\left(\pi g\left(y_{j}\right)\right)$. The pair $\left(y_{j}, K_{j}\right)$ describes that some eigenvalue $\lambda=\lambda_{j}(K)$ of $T_{1}$ on the right half plane converges to the point $i y_{j}$ on the imaginary axis as $K \rightarrow$ $K_{j}+0$. Since $\operatorname{Re}(\lambda)>0$, the eigenvalue $\lambda_{j}(K)$ is absorbed into the continuous spectrum on the imaginary axis and disappears at $K=K_{j}$. Suppose that $y_{c}$ satisfies $\sup _{j}\left\{g\left(y_{j}\right)\right\}=g\left(y_{c}\right)$ and put

$$
K_{c}=\inf _{j}\left\{K_{j}\right\}=\frac{2}{\pi g\left(y_{c}\right)} .
$$

In what follows, $\lambda_{c}(K)$ denotes the eigenvalue of $T_{1}$ satisfying $\lambda_{c} \rightarrow+0+i y_{c}$ as $K \rightarrow K_{c}+0$ ( $y_{c}$ and $\lambda_{c}$ may not be unique). The following formulae will be used later.

Lemma 3.3. The equalities

$$
\begin{aligned}
& D\left(i y_{c}\right):=\lim _{\lambda \rightarrow+0+i y_{c}} D(\lambda)=\frac{2}{K_{c}}, \\
& \left.\frac{d \lambda_{c}}{d K}\right|_{K=K_{c}}=\frac{-2}{K_{c}^{2} D^{\prime}\left(i y_{c}\right)}
\end{aligned}
$$

hold.

Proof. The first one follows from Eq.(3.9) and the definition of $\left(y_{c}, K_{c}\right)$. The derivative of Eq.(3.9) as a function of $\lambda$ gives

$$
D^{\prime}(\lambda)=\frac{-2}{i K(\lambda)^{2}} \frac{d K}{d \lambda}
$$

This proves the second one.

The eigenvalues of $T_{2}$ satisfy the same statement as Lemma 3.2. The limit $x \rightarrow+0$ for Eq.(3.8) provides

$$
\left\{\begin{array}{l}
\lim _{x \rightarrow+0} \int_{\mathbb{R}} \frac{x}{x^{2}+(y-2 \omega)^{2}} g(\omega) d \omega=\frac{1}{2} \pi g(y / 2)=\frac{h}{K}, \\
\lim _{x \rightarrow+0} \int_{\mathbb{R}} \frac{y-2 \omega}{x^{2}+(y-2 \omega)^{2}} g(\omega) d \omega=\frac{1}{2} \pi H[g](y / 2)=0 .
\end{array}\right.
$$


Let $y_{1}, y_{2}, \cdots$ be roots of the second equation, and define $K_{j}^{(2)}=2 h /\left(\pi g\left(y_{j} / 2\right)\right)$ and $K_{c}^{(2)}=\inf _{j}\left\{K_{j}^{(2)}\right\}$. In what follows, we assume the following;

(A1) $h<1$.

It is easy to verify that this condition is equivalent to $K_{c}<K_{c}^{(2)}$. This implies that the eigenvalue $\lambda_{c}$ of $T_{1}$ still exists on the right half plane after all eigenvalues of $T_{2}$ disappear as $K$ decreases. In other words, as $K$ increases from zero, the eigenvalue $\lambda_{c}$ of $T_{1}$ first emerges from the imaginary axis before some eigenvalue of $T_{2}$ emerges.

\section{Theorem 3.4 (Instability of the incoherent state).}

Suppose (A1) and $g(\omega)$ is continuous. If $0<K<K_{c}$, the spectra of operators $T_{1}, T_{2}, \cdots$ consist only of the continuous spectra on the imaginary axis. There exists a small number $\varepsilon>0$ such that when $K_{c}<K<K_{c}+\varepsilon$, the eigenvalue $\lambda_{c}$ of $T_{1}$ exists on the right half plane. Therefore, the incoherent state is linearly unstable.

This suggests that a first bifurcation occurs at $K=K_{c}$ and the eigenvalue $\lambda_{c}$ of $T_{1}$ plays an important role to the bifurcation.

Example 3.5. It is known that if $g(\omega)$ is an even and unimodal function, there exists a unique eigenvalue on the positive real axis for $K>K_{c}$. Since we are interested in a Hopf bifurcation in this paper, let us consider the following bimodal frequency distribution defined as the sum of two Lorentzian distribution [8]

$$
g(\omega)=\frac{1}{2 \pi}\left(\frac{1}{\left(\omega-\omega_{0}\right)^{2}+1}+\frac{1}{\left(\omega+\omega_{0}\right)^{2}+1}\right),
$$

where $\omega_{0}>0$ is a parameter. When $g^{\prime \prime}(0)>0 \Rightarrow \omega_{0} \geq 1 / \sqrt{3}$, it is a bimodal function. The equation $H[g](y)=0$ has at most three roots given by

$$
y_{1}=0, \quad y_{2}=\sqrt{\omega_{0}^{2}-1}, \quad y_{3}=-\sqrt{\omega_{0}^{2}-1} .
$$

Among them, $y_{2}$ and $y_{3}$ exist only when $\omega_{0}>1$. Otherwise, the eigenvalue uniquely exists on the positive real axis as in the unimodal distribution case. In what follows, we assume $\omega_{0}>1$. Since $g(0)<g\left(y_{2}\right)=g\left(y_{3}\right)=1 /(2 \pi), y_{c}$ and $K_{c}$ are given by

$$
y_{c}= \pm \sqrt{\omega_{0}^{2}-1}, \quad K_{c}=\frac{2}{\pi g\left(y_{c}\right)}=4
$$

Eq. (3.9) is calculated as

$$
D(\lambda)=\frac{\lambda+1}{(\lambda+1)^{2}+\omega_{0}^{2}}=\frac{2}{K} .
$$




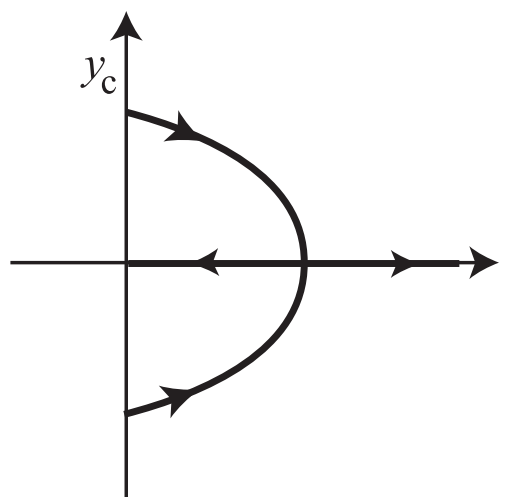

(a)



(b)

Figure 3: (a) The motion of the eigenvalues as $K$ increases for the distribution (3.11). The imaginary axis is the continuous spectrum. (b) The motion of the generalized eigenvalues as $K$ increases from zero for (3.11). The imaginary axis is a branch cut of the Riemann surface of the generalized resolvent. The dotted curve denotes the path of the generalized eigenvalue on the second Riemann sheet. See Section 5 for the detail.

This shows that there are at most two eigenvalues on the right half plane for each $K$. The motion of the eigenvalues $\lambda=\lambda(K)$ as $K$ increases is represented in Fig 3 (a). When $K<K_{c}=4$, there are no eigenvalues. At $K=K_{c}$, a pair of eigenvalues $\lambda_{c}\left(K_{c}\right)= \pm i \sqrt{\omega_{0}^{2}-1}$ pops up from the continuous spectrum on the imaginary axis. At $K=4 \omega_{0}>K_{c}$, two eigenvalues collide with one another on the real axis. For $K>4 \omega_{0}$, there are two eigenvalues on the positive real axis. One of them goes to the left side as $K$ increases, and it is absorbed into the continuous spectrum and disappears at $K=2 /(\pi g(0))>4 \omega_{0}$. The other goes to infinity on the positive real axis as $K \rightarrow \infty$. Later we will show that a Hopf bifurcation occurs at $K=K_{c}$.

\section{Linear stability}

When $0<K<K_{c}$, there are no spectra of operators $T_{1}, T_{2}, \cdots$ on the right half plane, while the continuous spectra of them exist on the imaginary axis. Hence, one may expect that the incoherent state is neutrally stable. Nevertheless, we will show that the order parameter is asymptotically stable in a certain sense. For this purpose, we need the following assumption. Let $\delta$ be a positive number and define the stripe region on $\mathbb{C}$

$$
S(\delta):=\{z \in \mathbb{C} \mid 0 \leq \operatorname{Im}(z) \leq \delta\} .
$$


We assume that

(A2) The distribution function $g(\omega)$ has an analytic continuation to the region $S(\delta)$. On $S(\delta)$, there exists a constant $C>0$ such that the estimate

$$
|g(z)| \leq \frac{C}{1+|z|^{2}}, \quad z \in S(\delta)
$$

holds.

Let $H_{+}$be the Hardy space on the upper half plane: the set of bounded holomorphic functions on the real axis and the upper half plane. It is a dense subspace of $L^{2}(\mathbb{R}, g(\omega) d \omega)$. For $\psi \in H_{+}$, set $\psi^{*}(z):=\overline{\psi(\bar{z})}$.

A function $f_{t} \in L^{2}(\mathbb{R}, g(\omega) d \omega)$ parameterized by $t$ is said to be convergent to zero in the weak sense if the inner product $\left(f_{t}, \psi^{*}\right)$ decays to zero as $t \rightarrow \infty$ for any $\psi \in H_{+}$. Note that $P_{0} \in H_{+}$and the order parameter is written as $\eta_{1}(t)=\left(Z_{1}, P_{0}\right)=\left(Z_{1}, P_{0}^{*}\right)$. This means that it is sufficient to consider the stability in the weak sense for the stability of the order parameter. The next lemma plays an important role in the generalized spectral theory.

Lemma 4.1. Let $f(z)$ be a holomorphic function on the region $S(\delta)$. Define a function $A[f](\lambda)$ of $\lambda$ to be

$$
A[f](\lambda)=\int_{\mathbb{R}} \frac{1}{\lambda-i \omega} f(\omega) d \omega
$$

for $\operatorname{Re}(\lambda)>0$. It has an analytic continuation $\hat{A}[f](\lambda)$ from the right half plane to the region $-\delta \leq \operatorname{Re}(\lambda) \leq 0$ given by

$$
\hat{A}[f](\lambda)= \begin{cases}A[f](\lambda) & \operatorname{Re}(\lambda)>0 \\ \lim _{\operatorname{Re}(\lambda) \rightarrow+0} A[f](\lambda) & \operatorname{Re}(\lambda)=0 \\ A[f](\lambda)+2 \pi f(-i \lambda) & -\delta \leq \operatorname{Re}(\lambda)<0 .\end{cases}
$$

See [2, 4] for the proof.

It is known that the semigroup $e^{T t}$ of an operator $T$ is expressed by the Laplace inversion formula

$$
e^{T t}=\lim _{y \rightarrow \infty} \frac{1}{2 \pi i} \int_{x-i y}^{x+i y} e^{\lambda t}(\lambda-T)^{-1} d \lambda,
$$

for $t>0$ (under a certain mild condition for $T[11]$ ). Here, $x>0$ is chosen so that the integral path is to the right of the spectrum of $T$ (see Fig, 4(a)). 
Lemma 4.2. The resolvent of $T_{1}=i \omega+i K f_{1} \mathcal{P}$ is given by

$$
\left(\lambda-T_{1}\right)^{-1} \phi=(\lambda-i \omega)^{-1} \phi+\frac{i K f_{1}}{1-i K f_{1} D(\lambda)}\left((\lambda-i \omega)^{-1} \phi, P_{0}\right) \frac{1}{\lambda-i \omega} .
$$

Let $\lambda_{c}$ be a simple eigenvalue of $T_{1}$. The projection $\Pi_{c}$ to the eigenspace of $\lambda_{c}$ is given by

$$
\Pi_{c} \phi=\frac{-1}{D^{\prime}\left(\lambda_{c}\right)}\left(\left(\lambda_{c}-i \omega\right)^{-1} \phi, P_{0}\right) \frac{1}{\lambda_{c}-i \omega}
$$

See [4] for the proof.

Lemma 4.2 provides

$$
\begin{aligned}
& \left(\left(\lambda-T_{1}\right)^{-1} \phi, \psi^{*}\right) \\
= & \left((\lambda-i \omega)^{-1} \phi, \psi^{*}\right)+\frac{i K f_{1}}{1-i K f_{1} D(\lambda)}\left((\lambda-i \omega)^{-1} \phi, P_{0}\right) \cdot\left((\lambda-i \omega)^{-1} \psi, P_{0}\right),
\end{aligned}
$$

which is meromorphic in $\lambda$ on the right half plane. Suppose $\phi, \psi \in H_{+}$. Due to Lemma 4.1, $\left(\left(\lambda-T_{1}\right)^{-1} \phi, \psi^{*}\right)$ has an analytic continuation, possibly with new singularities, to the region $-\delta \leq \operatorname{Re}(\lambda) \leq 0$ (Lemma 4.1 is applied to the factors $D(\lambda),\left((\lambda-i \omega)^{-1} \phi, \psi^{*}\right),\left((\lambda-i \omega)^{-1} \phi, P_{0}\right)$ and $\left.\left((\lambda-i \omega)^{-1} \psi, P_{0}\right)\right)$. A singularity on the left half plane is a root of the equation

$$
1-i K f_{1}(D(\lambda)+2 \pi g(-i \lambda))=0 .
$$

Such a singularity of the analytic continuation of the resolvent on the left half plane is called the generalized eigenvalue (see Sec.5 for the detail).

Now we can estimate the behavior of the semigroup by using the analytic continuation. We have

$$
\left(e^{T_{1} t} \phi, \psi^{*}\right)=\lim _{y \rightarrow \infty} \frac{1}{2 \pi i} \int_{x-i y}^{x+i y} e^{\lambda t}\left(\left(\lambda-T_{1}\right)^{-1} \phi, \psi^{*}\right) d \lambda
$$

where the integral path is given as in Fig 4 (a). When $\phi, \psi \in H_{+}$, the integrand $\left(\left(\lambda-T_{1}\right)^{-1} \phi, \psi^{*}\right)$ has an analytic continuation to the region $-\delta \leq$ $\operatorname{Re}(\lambda) \leq 0$ which is denoted by $\mathcal{R}(\lambda)$.

Lemma 4.3. Fix $K$ such that $0<K<K_{c}$. Take positive numbers $\varepsilon, R$ and consider the rectangle shaped closed path $C$ represented in Fig.4 (b). If $\varepsilon>0$ is sufficiently small, the analytic continuation of $\left(\left(\lambda-T_{1}\right)^{-1} \phi, \psi^{*}\right)$ is holomorphic inside $C$ for any $R>0$.

See [4] for the proof. 


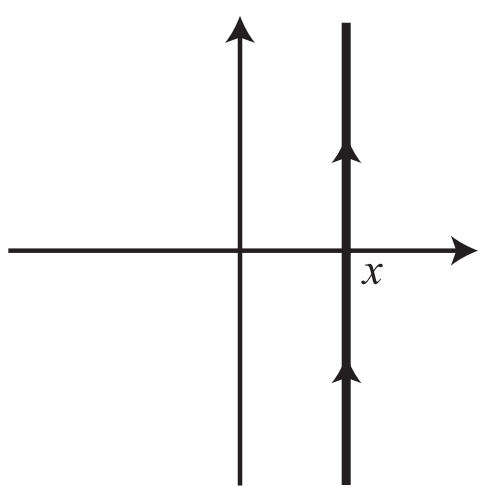

(a)

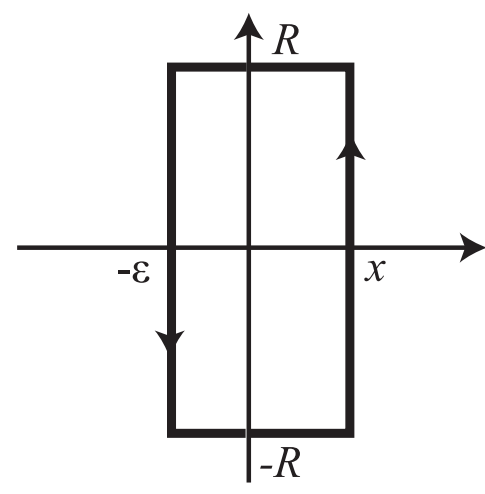

(b)

Figure 4: Deformation of the integral path for the Laplace inversion formula.

Because of this lemma, we have

$$
\begin{aligned}
0 & =\int_{x-i R}^{x+i R} e^{\lambda t}\left(\left(\lambda-T_{1}\right)^{-1} \phi, \psi^{*}\right) d \lambda+\int_{-\varepsilon+i R}^{-\varepsilon-i R} e^{\lambda t} \mathcal{R}(\lambda) d \lambda \\
& +\int_{x+i R}^{i R} e^{\lambda t}\left(\left(\lambda-T_{1}\right)^{-1} \phi, \psi^{*}\right) d \lambda+\int_{i R}^{i R-\varepsilon} e^{\lambda t} \mathcal{R}(\lambda) d \lambda \\
& +\int_{-i R}^{-i R+x} e^{\lambda t}\left(\left(\lambda-T_{1}\right)^{-1} \phi, \psi^{*}\right) d \lambda+\int_{-\varepsilon-i R}^{-i R} e^{\lambda t} \mathcal{R}(\lambda) d \lambda
\end{aligned}
$$

Due to the assumption (A2), we can verify that four integrals in the second and third lines above become zero as $R \rightarrow \infty$. Thus, we obtain

$$
\left(e^{T_{1} t} \phi, \psi^{*}\right)=\lim _{R \rightarrow \infty} \frac{1}{2 \pi i} \int_{-\varepsilon-i R}^{-\varepsilon+i R} e^{\lambda t} \mathcal{R}(\lambda) d \lambda .
$$

This proves $\left|\left(e^{T_{1} t} \phi, \psi^{*}\right)\right| \sim O\left(e^{-\varepsilon t}\right)$ as $t \rightarrow \infty$. We can show the same result for the operators $T_{2}, T_{3}, \cdots$.

Theorem 4.4 (Local stability of the incoherent state).

Suppose (A1) and (A2). When $0<K<K_{c}$, $\left(e^{T_{j} t} \phi, \psi^{*}\right)$ decays to zero exponentially as $t \rightarrow \infty$ for any $j=1,2, \cdots$ and any $\phi, \psi \in H_{+}$. Thus, the incoherent state is linearly asymptotically stable in the weak sense.

\section{The generalized spectral theory}

For the study of a bifurcation, we need generalized spectral theory developed in [3] and applied to the Kuramoto model in [2] because the operator $T_{1}$ has 
the continuous spectrum on the imaginary axis (thus, the standard center manifold reduction is not applicable). In this section, a simple review of the generalized spectral theory is given. All proofs are included in [2, 3].

Let $H_{+}$be the Hardy space on the upper half plane with the norm

$$
\|\phi\|_{H_{+}}=\sup _{\operatorname{Im}(z)>0}|\phi(z)| .
$$

With this norm, $H_{+}$is a Banach space. Let $H_{+}^{\prime}$ be the dual space of $H_{+}$; the set of continuous anti-linear functionals on $H_{+}$. For $\mu \in H_{+}^{\prime}$ and $\phi \in H_{+}$, $\mu(\phi)$ is denoted by $\langle\mu \mid \phi\rangle$. For any $a, b \in \mathbb{C}, \phi, \psi \in H_{+}$and $\mu, \xi \in H_{+}^{\prime}$, the equalities

$$
\begin{aligned}
& \langle\mu \mid a \phi+b \psi\rangle=\bar{a}\langle\mu \mid \phi\rangle+\bar{b}\langle\mu \mid \psi\rangle, \\
& \langle a \mu+b \xi \mid \phi\rangle=a\langle\mu \mid \phi\rangle+b\langle\xi \mid \phi\rangle
\end{aligned}
$$

hold. An element of $H_{+}^{\prime}$ is called a generalized function. The space $H_{+}$is a dense subspace of $L^{2}=L^{2}(\mathbb{R}, g(\omega) d \omega)$ and the embedding $H_{+} \hookrightarrow L^{2}$ is continuous. Then, we can show that the dual $\left(L^{2}\right)^{\prime}$ of $L^{2}$ is dense in $H_{+}^{\prime}$ and it is continuously embedded in $H_{+}^{\prime}$. Since $L^{2}$ is a Hilbert space satisfying $\left(L^{2}\right)^{\prime} \simeq L^{2}$, we have three topological vector spaces called a Gelfand triplet

$$
H_{+} \subset L^{2}(\mathbb{R}, g(\omega) d \omega) \subset H_{+}^{\prime} .
$$

If an element $\phi \in H_{+}^{\prime}$ is included in $L^{2}(\mathbb{R}, g(\omega) d \omega)$, then $\langle\phi \mid \psi\rangle$ is given by

$$
\langle\phi \mid \psi\rangle:=\left(\phi, \psi^{*}\right)=\int_{\mathbb{R}} \phi(\omega) \psi(\omega) g(\omega) d \omega .
$$

(the conjugate $\psi^{*}$ is introduced to avoid the complex conjugate $\overline{\psi(\omega)}$ in the integrand). Our operator $T_{1}$ and the above triplet satisfy all assumptions given in [3] to develop a generalized spectral theory. Now we give a brief review of the theory. In what follows, we assume (A2).

The multiplication operator $\phi \mapsto i \omega \phi$ has the continuous spectrum on the imaginary axis; its resolvent is given by $(\lambda-i \omega)^{-1}$, and it is not included in $L^{2}(\mathbb{R}, g(\omega) d \omega)$ when $\lambda$ is a purely imaginary number. Nevertheless, we show that the resolvent has an analytic continuation from the right half plane to the left half plane in the generalized sense. We define an operator $A(\lambda): H_{+} \rightarrow H_{+}^{\prime}$, parameterized by $\lambda \in \mathbb{C}$, to be

$$
\langle A(\lambda) \phi \mid \psi\rangle= \begin{cases}\left((\lambda-i \omega)^{-1} \phi, \psi^{*}\right), & \operatorname{Re}(\lambda)>0, \\ \lim _{\operatorname{Re}(\lambda) \rightarrow+0}\left((\lambda-i \omega)^{-1} \phi, \psi^{*}\right) & \operatorname{Re}(\lambda)=0, \\ \left((\lambda-i \omega)^{-1} \phi, \psi^{*}\right) & \\ +2 \pi \phi(-i \lambda) \psi(-i \lambda) g(-i \lambda) & -\delta \leq \operatorname{Re}(\lambda)<0,\end{cases}
$$


for $\phi, \psi \in H_{+}$. Due to Lemma 4.1, $\langle A(\lambda) \phi \mid \psi\rangle$ is holomorphic. That is, $A(\lambda) \phi$ is a $H_{+}^{\prime}$-valued holomorphic function in $\lambda$. In particular, $A(\lambda)$ coincides with $(\lambda-i \omega)^{-1}$ when $\operatorname{Re}(\lambda)>0$. Since the continuous spectrum of the multiplication operator by $i \omega$ is the whole imaginary axis, $(\lambda-i \omega)^{-1}$ does not have an analytic continuation from the right half plane to the left half plane as an operator on $L^{2}(\mathbb{R}, g(\omega) d \omega)$, however, it has a continuation $A(\lambda)$ if it is regarded as an operator from $H_{+}$to $H_{+}^{\prime} . A(\lambda)$ is called the generalized resolvent of the multiplication operator by $i \omega$.

The next purpose is to define an analytic continuation of the resolvent of $T_{1}$ in the generalized sense. Note that $\left(\lambda-T_{1}\right)^{-1}$ is rearranged as

$$
\left(\lambda-i \omega-i K f_{1} \mathcal{P}\right)^{-1}=(\lambda-i \omega)^{-1} \circ\left(\mathrm{id}-i K f_{1} \mathcal{P}(\lambda-i \omega)^{-1}\right)^{-1} .
$$

Since the analytic continuation of $(\lambda-i \omega)^{-1}$ in the generalized sense is $A(\lambda)$, we define the generalized resolvent $\mathcal{R}(\lambda): H_{+} \rightarrow H_{+}^{\prime}$ of $T_{1}$ by

$$
\mathcal{R}(\lambda):=A(\lambda) \circ\left(\mathrm{id}-i K f_{1} \mathcal{P}^{\times} A(\lambda)\right)^{-1},
$$

where $\mathcal{P}^{\times}: H_{+}^{\prime} \rightarrow H_{+}^{\prime}$ is the dual operator of $\mathcal{P}$. For each $\phi \in H_{+}, \mathcal{R}(\lambda) \phi$ is a $H_{+}^{\prime}$-valued meromorphic function. It is easy to verify that when $\operatorname{Re}(\lambda)>0$, it is reduced to the usual resolvent $\left(\lambda-T_{1}\right)^{-1}$. Thus, $\mathcal{R}(\lambda)$ gives a meromorphic continuation of $\left(\lambda-T_{1}\right)^{-1}$ from the right half plane to the left half plane as a $H_{+}^{\prime}$-valued operator. Again, note that $T_{1}$ has the continuous spectrum on the imaginary axis, so that it has no continuation as an operator on $L^{2}(\mathbb{R}, g(\omega) d \omega)$.

A generalized eigenvalue is defined as a singularity of $\mathcal{R}(\lambda)$, namely a singularity of $\left(\mathrm{id}-i K f_{1} \mathcal{P}^{\times} A(\lambda)\right)^{-1}$.

Definition 5.1. If the equation

$$
\left(\mathrm{id}-i K f_{1} \mathcal{P}^{\times} A(\lambda)\right) \mu=0
$$

has a nonzero solution $\mu$ in $H_{+}^{\prime}$ for some $\lambda \in \mathbb{C}, \lambda$ is called a generalized eigenvalue and $\mu$ is called a generalized eigenfunction.

It is easy to verify that this equation is equivalent to

$$
\frac{2}{K}= \begin{cases}D(\lambda) & \operatorname{Re}(\lambda)>0 \\ \lim _{\operatorname{Re}(\lambda) \rightarrow+0} D(\lambda) & \operatorname{Re}(\lambda)=0 \\ D(\lambda)+2 \pi g(-i \lambda) & -\delta \leq \operatorname{Re}(\lambda)<0\end{cases}
$$

where we use $f_{1}=1 /(2 i)$. When $\operatorname{Re}(\lambda)>0$, this is reduced to Eq.(3.9). In this case, $\mu$ is included in $L^{2}(\mathbb{R}, g(\omega) d \omega)$ and a generalized eigenvalue on the right half plane is an eigenvalue in the usual sense. When $\operatorname{Re}(\lambda) \leq 0$, 
this equation is equivalent to Eq.(4.6). The associated generalized eigenfunction is not included in $L^{2}(\mathbb{R}, g(\omega) d \omega)$ but an element of the dual space $H_{+}^{\prime}$. Although a generalized eigenvalue is not a true eigenvalue of $T_{1}$, it is an eigenvalue of the dual operator:

Theorem 5.2 [2, 3]. Let $\lambda$ and $\mu$ be a generalized eigenvalue and the associated generalized eigenfunction. The equality $T_{1}^{\times} \mu=\lambda \mu$ holds.

Let $\lambda_{0}$ be a generalized eigenvalue of $T_{1}$ and $\gamma_{0}$ a small simple closed curve enclosing $\lambda_{0}$. The generalized Riesz projection $\Pi_{0}: H_{+} \rightarrow H_{+}^{\prime}$ is defined by

$$
\Pi_{0}=\frac{1}{2 \pi i} \int_{\gamma_{0}} \mathcal{R}(\lambda) d \lambda .
$$

As in the usual spectral theory, the image of it gives the generalized eigenspace associated with $\lambda_{0}$.

Let $\lambda=\lambda_{c}(K)$ be an eigenvalue of $T_{1}$ defined in Sec.3. Recall that when $K_{c}<K, \lambda_{c}$ exists on the right half plane. As $K$ decreases, $\lambda_{c}$ goes to the left side, and at $K=K_{c}, \lambda_{c}$ is absorbed into the continuous spectrum on the imaginary axis and disappears. However, we can show that even for $0<K<K_{c}, \lambda_{c}$ remains to exist as a root of Eq.(5.3) because the right hand side of Eq. (5.3) is holomorphic. This means that although $\lambda_{c}$ disappears from the original complex plane at $K=K_{c}$, it still exists for $0<K<K_{c}$ as a generalized eigenvalue on the Riemann surface of the generalized resolvent $\mathcal{R}(\lambda)$. In the generalized spectral theory, the resolvent $\left(\lambda-T_{1}\right)^{-1}$ is regarded as an operator from $H_{+}$to $H_{+}^{\prime}$, not on $L^{2}(\mathbb{R}, g(\omega) d \omega)$. Then, it has an analytic continuation from the right half plane to the left half plane as $H_{+}^{\prime}-$ valued operator. The continuous spectrum on the imaginary axis becomes a branch cut of the Riemann surface of the resolvent. On the Riemann surface, the left half plane is two-sheeted (see Fig!5). We call a singularity of the generalized resolvent on the second Riemann sheet the generalized eigenvalue.

On the dual space $H_{+}^{\prime}$, the weak dual topology is equipped; a sequence $\left\{\mu_{n}\right\} \subset H_{+}^{\prime}$ is said to be convergent to $\mu \in H_{+}^{\prime}$ if $\left\langle\mu_{n} \mid \psi\right\rangle \in \mathbb{C}$ is convergent to $\langle\mu \mid \psi\rangle$ for each $\psi \in H_{+}$. Recall that an eigenfunction of a usual eigenvalue $\lambda$ of $T_{1}$ is given by $v_{\lambda}(\omega)=(\lambda-i \omega)^{-1}$ (Eq.(3.6) $)$. A generalized eigenfunction $\mu_{\lambda}$ of a generalized eigenvalue $i y$ on the imaginary axis is given by

$$
\mu_{\lambda}=\lim _{\lambda \rightarrow+0+i y} \frac{1}{\lambda-i \omega}
$$

where the limit is considered with respect to the weak dual topology. This means that $\left\langle\mu_{\lambda} \mid \psi\right\rangle$ is defined by

$$
\left\langle\mu_{\lambda} \mid \psi\right\rangle=\lim _{\lambda \rightarrow+0+i y}\left\langle\frac{1}{\lambda-i \omega} \mid \psi\right\rangle=\lim _{\lambda \rightarrow+0+i y} \int_{\mathbb{R}} \frac{1}{\lambda-i \omega} \psi(\omega) g(\omega) d \omega .
$$




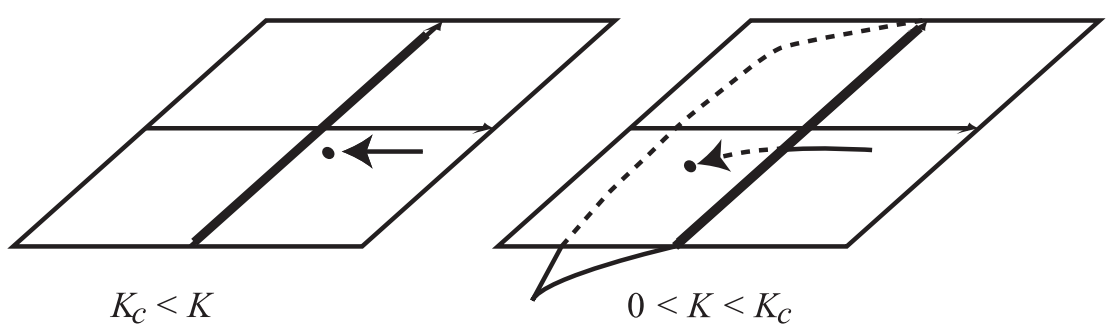

Figure 5: The motion of the (generalized) eigenvalue as $K$ decreases. When $0<K<K_{c}$, it lies on the second Riemann sheet of the resolvent and it is not a usual eigenvalue but a generalized eigenvalue.

A generalized eigenfunction $\mu_{\lambda}$ associated with a generalized eigenvalue $\lambda$ on the left half plane is given by

$$
\left\langle\mu_{\lambda} \mid \psi\right\rangle=\int_{\mathbb{R}} \frac{1}{\lambda-i \omega} \psi(\omega) g(\omega) d \omega+2 \pi \psi(-i \lambda) g(-i \lambda) .
$$

To perform a center manifold reduction, we need the definition of a center subspace. Usually, it is defined to be an eigenspace associated with eigenvalues on the imaginary axis. For our case, the operators $T_{1}, T_{2}, \cdots$ have the continuous spectra on the imaginary axis. Therefore, we define a generalized center subspace as a space spanned by generalized eigenfunctions associated with generalized eigenvalues on the imaginary axis. Note that this is a subspace of the dual $H_{+}^{\prime}$, not of $L^{2}(\mathbb{R}, g(\omega) d \omega)$. As $K$ increases from zero, some of the generalized eigenvalues of $T_{1}$ get across the imaginary axis at $K=K_{c}$, and they become usual eigenvalues on the right half plane (see Fig 5 ). Hence, there is a nontrivial generalized center subspace at $K=K_{c}$ given by

$$
\mathbf{E}^{c}:=\operatorname{span}\left\{\mu_{\lambda} \mid \lambda\left(K_{c}\right) \in i \mathbb{R}\right\}
$$

The next purpose is to perform a center manifold reduction.

Example 5.3. Let us consider the distribution (3.11) given in Example 3.5. The equation (5.3) for generalized eigenvalues is given by (3.12); the left hand side of it already gives an analytic continuation of $D(\lambda)$. By solving it, it turns out that two generalized eigenvalues exist at $\lambda(0)=-1 \pm i \omega_{0}$ when $K=0$. As $K$ increases, they go to the right side as is shown in Fig. [3 (b). They get across the imaginary axis when $K=K_{c}=4$, and become usual eigenvalues for $K>K_{c}$. One of them again becomes a generalized eigenvalue at $K=2 /(\pi g(0))$ by getting across the imaginary axis from the right to the left. The generalized center subspace for $K=K_{c}$ is a two-dimensional space. 


\section{Center manifold reduction}

Recall that $y_{c} \in \mathbb{R}$ is defined as a number satisfying $\sup _{j}\left\{g\left(y_{j}\right)\right\}=g\left(y_{c}\right)$, where $y_{1}, y_{2}, \cdots$ are roots of the equation $H[g](y)=0$. This gives a point $i y_{c}$ on the imaginary axis to which some eigenvalue of $T_{1}$ approaches as $K \rightarrow K_{c}+0$. For a Hopf bifurcation, we assume the following:

(A3) There are exactly two nonzero values $y_{c}$ and $-y_{c}$ satisfying $\sup _{j}\left\{g\left(y_{j}\right)\right\}=$ $g\left( \pm y_{c}\right)$. Each of the corresponding eigenvalue of $T_{1}$ denoted by $\lambda_{c}^{+}(K)$ and $\lambda_{c}^{-}(K)$, respectively, is simple near $K_{c}$ (i.e. the eigenspace is one dimensional).

(A4) The real part of $\left.\frac{d \lambda_{c}^{ \pm}}{d K}\right|_{K=K_{c}}$ is positive.

(A5) $g(\omega)$ is an even function.

The assumption (A3) implies that the generalized center subspace at $K=K_{c}$ is a two dimensional space given by

$$
\mathbf{E}^{c}=\operatorname{span}\left\{\mu_{+}, \mu_{-}\right\}, \quad \mu_{ \pm}:=\lim _{\lambda \rightarrow+0 \pm i y_{c}} \frac{1}{\lambda-i \omega} .
$$

The assumption (A4) means that the generalized eigenvalues $\lambda_{c}^{ \pm}$of $T_{1}$ transversely get across the imaginary axis from the left to the right. Due to (A5), it is easy to verify that the following equalities hold:

$$
D\left(i y_{c}\right)=D\left(-i y_{c}\right)=\frac{2}{K_{c}}, D^{\prime}\left(i y_{c}\right)=\overline{D^{\prime}\left(-i y_{c}\right)}, \quad D^{\prime \prime}\left(i y_{c}\right)=\overline{D^{\prime \prime}\left(-i y_{c}\right)} .
$$

It seems that (A3) and (A4) are satisfied for a wide class of even and bimodal distributions $g(\omega)$ as long as the distance of two peaks are sufficiently far apart, see Example 3.5.

In what follows, we assume (A1) to (A5). We expect that a Hopf bifurcation occurs at $K=K_{c}$. In Chiba [2], the existence of the one dimensional center manifold in $H_{+}^{\prime}$ is proved for the Kuramoto model when $g(\omega)$ is even and unimodal. In this paper, we formally perform the center manifold reduction without a proof of the existence of a center manifold.

We put $\varepsilon=K-K_{c}$, which plays a role of a bifurcation parameter. Our ingredients are;

Equations: The equations (2.3) for $j=1,2$ with $f_{1}=1 /(2 i), f_{2}=h /(2 i)$ are given by

$$
\left\{\begin{array}{l}
\dot{Z}_{1}=T_{c} Z_{1}+\frac{\varepsilon}{2} \mathcal{P} Z_{1}+\frac{K}{2}\left(h \cdot \eta_{2} Z_{-1}-\overline{\eta_{1}} Z_{2}-h \cdot \overline{\eta_{2}} Z_{3}\right), \\
\dot{Z}_{2}=T_{2} Z_{2}+K\left(\eta_{1} Z_{1}-\overline{\eta_{1}} Z_{3}-h \cdot \overline{\eta_{2}} Z_{4}\right),
\end{array}\right.
$$


where $T_{c}$ is an operator $T_{1}$ estimated at $K=K_{c}$; that is, $K$ in $T_{1}$ is denoted by $K=K_{c}+\varepsilon$ and accordingly $T_{1}=T_{c}+\varepsilon \mathcal{P} / 2$.

Center subspace: As $K$ increases from zero, a pair of the generalized eigenvalues of $T_{1}$ denoted by $\lambda_{c}^{ \pm}(K)$ gets across the imaginary axis at $\pm i y_{c}$ when $K=K_{c}$, and they become usual eigenvalues on the right half plane when $K>K_{c}$. The associated generalized eigenfunctions at $K=K_{c}$ and the generalized center subspace is given in (6.1).

Projection: The projection to an eigenspace is given in Lemma 4.2. The projection to the generalized center subspace spanned by $\mu_{+}$and $\mu_{-}$is

$\Pi_{c} \phi=\frac{-1}{D^{\prime}\left(i y_{c}\right)} \lim _{\lambda \rightarrow i y_{c}}\left((\lambda-i \omega)^{-1} \phi, P_{0}\right) \mu_{+}+\frac{-1}{D^{\prime}\left(-i y_{c}\right)} \lim _{\lambda \rightarrow-i y_{c}}\left((\lambda-i \omega)^{-1} \phi, P_{0}\right) \mu_{-}$.

We divide our result into two cases, $h=0$ and $h \neq 0$ because types of bifurcations of them are different.

\subsection{Center manifold reduction $(h=0)$}

Assume $h=0$. Then, $T_{2}=2 i \omega$. Since $\Pi_{c} Z_{1}$ is a linear combination of $\mu_{+}$ and $\mu_{-}$, we suppose $\Pi_{c} Z_{1}=K_{c} / 2 \cdot\left(\alpha_{+}(t) \mu_{+}+\alpha_{-}(t) \mu_{-}\right)$. The scalar valued functions $\alpha_{+}(t)$ and $\alpha_{-}(t)$ denote coordinates on the center subspace, and our purpose is to derive the dynamics of $\alpha_{ \pm}$. Since a solution decays to zero with an exponential rate for $\left(\mathrm{id}-\Pi_{c}\right) Z_{1}$ direction and $Z_{j}(j=2,3, \cdots)$ directions, we assume that $\left(\mathrm{id}-\Pi_{c}\right) Z_{1}$ and $Z_{j}(j=2,3, \cdots)$ are of order $O\left(\alpha^{2}\right)$ which stand for $O\left(\alpha_{+}^{2}, \alpha_{+} \alpha_{-}, \alpha_{-}^{2}\right)$. Thus, we write

$$
Z_{1}=\frac{K_{c}}{2}\left(\alpha_{+}(t) \mu_{+}+\alpha_{-}(t) \mu_{-}\right)+O\left(\alpha^{2}\right)
$$

Then, $\eta_{1}$ is given by

$$
\begin{aligned}
\eta_{1} & =\int_{\mathbb{R}} Z_{1} \cdot g(\omega) d \omega \\
& =\frac{K_{c}}{2} \alpha_{+} \lim _{\lambda \rightarrow i y_{c}} \int_{\mathbb{R}} \frac{1}{\lambda-i \omega} g(\omega) d \omega+\frac{K_{c}}{2} \alpha_{-} \lim _{\lambda \rightarrow-i y_{c}} \int_{\mathbb{R}} \frac{1}{\lambda-i \omega} g(\omega) d \omega+O\left(\alpha^{2}\right) \\
& =\alpha_{+}+\alpha_{-}+O\left(\alpha^{2}\right),
\end{aligned}
$$

where we have used Eq.(6.2). Further, we make the following ansatz

$$
\varepsilon \sim O\left(\alpha^{2}\right), \quad \frac{d \alpha_{ \pm}}{d t}= \pm i y_{c} \alpha_{ \pm}+O\left(\alpha^{2}\right)
$$

which will be verified if the dynamics on the center manifold is derived. 
For $m, n=0,1,2, \cdots$, we define functionals denoted by $\mu_{+}^{m} \cdot \mu_{-}^{n} \in H_{+}^{\prime}$ by

$$
\mu_{+}^{m} \cdot \mu_{-}^{n}:=\lim _{\lambda_{+} \rightarrow+i y_{c} \lambda_{-} \rightarrow-i y_{c}} \lim _{\left(\lambda_{+}-i \omega\right)^{m}} \frac{1}{\left(\lambda_{-}-i \omega\right)^{n}}
$$

where the limit is considered with respect to the weak dual topology.

Lemma 6.1. The following equalities hold.

$$
\begin{aligned}
& \omega \mu_{ \pm}^{2}=i \mu_{ \pm} \pm y_{c} \mu_{ \pm}^{2}, \\
& \omega \mu_{ \pm}=i \pm y_{c} \mu_{ \pm}, \\
& \left\langle\mu_{ \pm} P_{0} \mid P_{0}\right\rangle=D\left( \pm i y_{c}\right)=\frac{2}{K_{c}}, \\
& \left\langle\mu_{ \pm}^{2} P_{0} \mid P_{0}\right\rangle=-D^{\prime}\left( \pm i y_{c}\right), \\
& \left\langle\mu_{ \pm}^{3} P_{0} \mid P_{0}\right\rangle=\frac{1}{2} D^{\prime \prime}\left( \pm i y_{c}\right), \\
& \left\langle\mu_{+} \cdot \mu_{-} P_{0} \mid P_{0}\right\rangle=0, \\
& \left\langle\mu_{+}^{2} \cdot \mu_{-} P_{0} \mid P_{0}\right\rangle=\frac{1}{2 i y_{c}} D^{\prime}\left(i y_{c}\right), \\
& \left\langle\mu_{+} \cdot \mu_{-}^{2} P_{0} \mid P_{0}\right\rangle=\frac{-1}{2 i y_{c}} D^{\prime}\left(-i y_{c}\right),
\end{aligned}
$$

where $P_{0}(\omega)=1$ is a constant function.

Proof. For the first equality, we have

$$
\begin{aligned}
\omega \mu_{ \pm}^{2} & =\lim _{\lambda \rightarrow \pm i y_{c}} \frac{\omega}{(\lambda-i \omega)^{2}}=i \cdot \lim _{\lambda \rightarrow \pm i y_{c}} \frac{(\lambda-i \omega)-\lambda}{(\lambda-i \omega)^{2}} \\
& =i \cdot \lim _{\lambda \rightarrow \pm i y_{c}} \frac{1}{\lambda-i \omega} \pm y_{c} \lim _{\lambda \rightarrow \pm i y_{c}} \frac{1}{(\lambda-i \omega)^{2}} \\
& =i \mu_{ \pm} \pm y_{c} \mu_{ \pm}^{2} .
\end{aligned}
$$

The second one is proved in a similar manner. The third one is given in Lemma 3.3. The fourth and fifth equalities are easily shown by the integration by parts, see Lemma 3.1. To prove the sixth equality, we use the partial fraction decomposition as

$$
\begin{aligned}
\left\langle\mu_{+} \cdot \mu_{-} P_{0} \mid P_{0}\right\rangle & =\lim _{\lambda_{+} \rightarrow+i y_{c} \lambda_{-} \rightarrow-i y_{c}} \lim _{\mathbb{R}} \frac{1}{\left(\lambda_{+}-i \omega\right)\left(\lambda_{-}-i \omega\right)} g(\omega) d \omega \\
& =\lim _{\lambda_{+} \rightarrow+i y_{c} \lambda_{-} \rightarrow-i y_{c}} \frac{1}{\lambda_{+}-\lambda_{-}} \int_{\mathbb{R}}\left(\frac{-1}{\lambda_{+}-i \omega}+\frac{1}{\lambda_{-}-i \omega}\right) g(\omega) d \omega \\
& =\frac{1}{2 i y_{c}}\left(-D\left(i y_{c}\right)+D\left(-i y_{c}\right)\right)=0 .
\end{aligned}
$$


The last two equalities are also verified by the partial fraction decomposition.

Lemma 6.2. Define

$$
Z_{2}=\frac{K_{c}^{2}}{4} \alpha_{+}^{2} \mu_{+}^{2}+\frac{K_{c}^{2}}{4} \alpha_{-}^{2} \mu_{-}^{2}-\frac{K_{c}^{2}}{4 i y_{c}} \alpha_{+} \alpha_{-}\left(\mu_{+}-\mu_{-}\right)+O\left(\alpha^{3}\right)
$$

It satisfies the second differential equation of (6.3) up to the order $O\left(\alpha^{3}\right)$.

Proof. By substituting Eqs.(6.5), (6.6) and (6.9) in the equation, we can confirm with the aid of Lemma 6.1 and (6.7) that

$$
\dot{Z}_{2}-\left(T_{2} Z_{2}+K\left(\eta_{1} Z_{1}-\overline{\eta_{1}} Z_{3}\right)\right)
$$

is of order $O\left(\alpha^{3}\right)$.

Let us apply the projection $\Pi_{c}$ to the both sides of the first equation of Eq.(6.3) to get

$$
\frac{K_{c}}{2}\left(\dot{\alpha}_{+} \mu_{+}+\dot{\alpha}_{-} \mu_{-}\right)=T_{c}^{\times} \Pi_{c} Z_{1}+\frac{\varepsilon}{2} \eta_{1} \Pi_{c} P_{0}-\frac{K}{2} \bar{\eta}_{1} \Pi_{c} Z_{2} .
$$

Theorem 5.2 gives

$$
T_{c}^{\times} \Pi_{c} Z_{1}=\frac{K_{c}}{2} T_{c}^{\times}\left(\alpha_{+} \mu_{+}+\alpha_{-} \mu_{-}\right)=\frac{K_{c}}{2} \cdot i y_{c} \cdot\left(\alpha_{+} \mu_{+}-\alpha_{-} \mu_{-}\right) .
$$

The definition of $\Pi_{c}$ combined with Lemma 6.1 yields

$$
\begin{aligned}
\Pi_{c} P_{0}= & \frac{-2}{K_{c} D^{\prime}\left(i y_{c}\right)} \mu_{+}+\frac{-2}{K_{c} D^{\prime}\left(-i y_{c}\right)} \mu_{-}, \\
\Pi_{c} Z_{2}= & \frac{-K_{c}^{2}}{4 D^{\prime}\left(i y_{c}\right)}\left(\frac{1}{2} \alpha_{+}^{2} D^{\prime \prime}\left(i y_{c}\right)-\frac{1}{2 i y_{c}} \alpha_{-}^{2} D^{\prime}\left(-i y_{c}\right)+\frac{1}{i y_{c}} \alpha_{+} \alpha_{-} D^{\prime}\left(i y_{c}\right)\right) \mu_{+} \\
& +\frac{-K_{c}^{2}}{4 D^{\prime}\left(-i y_{c}\right)}\left(\frac{1}{2} \alpha_{-}^{2} D^{\prime \prime}\left(-i y_{c}\right)+\frac{1}{2 i y_{c}} \alpha_{+}^{2} D^{\prime}\left(i y_{c}\right)-\frac{1}{i y_{c}} \alpha_{+} \alpha_{-} D^{\prime}\left(-i y_{c}\right)\right) \mu_{-} \\
& +O\left(\alpha^{3}\right) .
\end{aligned}
$$

Substituting these equalities into Eq. (6.10) and comparing the coefficients of $\mu_{+}$and $\mu_{-}$, respectively, in the both sides of the equation, we obtain the dynamics on the center manifold

$$
\left\{\begin{array}{l}
\frac{d \alpha_{+}}{d t}=i y_{c} \alpha_{+}+p_{1} \varepsilon\left(\alpha_{+}+\alpha_{-}\right)+\left(\bar{\alpha}_{+}+\bar{\alpha}_{-}\right)\left(p_{2} \alpha_{+}^{2}+p_{3} \alpha_{-}^{2}+p_{4} \alpha_{+} \alpha_{-}\right)+O\left(\alpha^{4}\right), \\
\frac{d \alpha_{-}}{d t}=-i y_{c} \alpha_{-}+\bar{p}_{1} \varepsilon\left(\alpha_{+}+\alpha_{-}\right)+\left(\bar{\alpha}_{+}+\bar{\alpha}_{-}\right)\left(\bar{p}_{2} \alpha_{-}^{2}+\bar{p}_{3} \alpha_{+}^{2}+\bar{p}_{4} \alpha_{+} \alpha_{-}\right)+O\left(\alpha^{4}\right),
\end{array}\right.
$$


where $p_{1}$ to $p_{4}$ are complex numbers defined by

$$
p_{1}=\frac{-2}{K_{c}^{2} D^{\prime}\left(i y_{c}\right)}, \quad p_{2}=\frac{K_{c}^{2} D^{\prime \prime}\left(i y_{c}\right)}{8 D^{\prime}\left(i y_{c}\right)}, \quad p_{3}=-\frac{K_{c}^{2} \overline{D^{\prime}\left(i y_{c}\right)}}{8 i y_{c} D^{\prime}\left(i y_{c}\right)}, \quad p_{4}=\frac{K_{c}^{2}}{4 i y_{c}} .
$$

This is a (real) four dimensional dynamical system. The next purpose is to reduce it. Since the system is invariant under the action $\left(\alpha_{+}, \alpha_{-}\right) \mapsto$ $\left(e^{i \beta} \alpha_{+}, e^{i \beta} \alpha_{-}\right)$for $\beta \in \mathbb{R}$, we can assume without loss of generality that $\arg \left(\alpha_{+}\right)+\arg \left(\alpha_{-}\right)=0$. Hence, we assume $\alpha_{ \pm}=r_{ \pm} e^{ \pm i \psi}$ with $r_{ \pm}, \psi \in \mathbb{R}$. Substituting this into the system, we obtain the three dimensional system

$$
\left\{\begin{array}{l}
\dot{\psi}=y_{c}+O\left(r_{ \pm}^{2}\right)=y_{c}+O(\varepsilon), \\
\dot{r}_{+}=\varepsilon \operatorname{Re}\left(p_{1}\right) r_{+}+\varepsilon \operatorname{Re}\left(p_{1} e^{-2 i \psi}\right) r_{-}+\operatorname{Re}\left(p_{2}\right) r_{+}^{3}+\operatorname{Re}\left(p_{2} e^{2 i \psi}\right) r_{+}^{2} r_{-} \\
\quad+\operatorname{Re}\left(p_{3} e^{-4 i \psi}\right) r_{+} r_{-}^{2}+\operatorname{Re}\left(p_{3} e^{-2 i \psi}\right) r_{-}^{3}+\operatorname{Re}\left(p_{4} e^{-2 i \psi}\right) r_{+}^{2} r_{-}+O\left(r_{ \pm}^{4}\right), \\
\dot{r}_{-}=\varepsilon \operatorname{Re}\left(p_{1}\right) r_{-}+\varepsilon \operatorname{Re}\left(p_{1} e^{-2 i \psi}\right) r_{+}+\operatorname{Re}\left(p_{2}\right) r_{-}^{3}+\operatorname{Re}\left(p_{2} e^{2 i \psi}\right) r_{+} r_{-}^{2} \\
\quad+\operatorname{Re}\left(p_{3} e^{-4 i \psi}\right) r_{+}^{2} r_{-}+\operatorname{Re}\left(p_{3} e^{-2 i \psi}\right) r_{+}^{3}+\operatorname{Re}\left(p_{4} e^{-2 i \psi}\right) r_{+} r_{-}^{2}+O\left(r_{ \pm}^{4}\right) .
\end{array}\right.
$$

To derive this, note that $\operatorname{Re}\left(p_{4}\right)=0$. Now we apply the averaging method. The right hand sides of the equations of $r_{+}$and $r_{-}$are averaged over $\psi$ to obtain the averaging equation

$$
\left\{\begin{array}{l}
\dot{r}_{+}=\varepsilon \operatorname{Re}\left(p_{1}\right) r_{+}+\operatorname{Re}\left(p_{2}\right) r_{+}^{3}+O\left(r_{ \pm}^{4}\right) \\
\dot{r}_{-}=\varepsilon \operatorname{Re}\left(p_{1}\right) r_{-}+\operatorname{Re}\left(p_{2}\right) r_{-}^{3}+O\left(r_{ \pm}^{4}\right) .
\end{array}\right.
$$

It is known that the averaging equation provides an approximate solution within the error of order $O(\varepsilon)$. Further, if the averaging equation has a stable fixed point, then the original system has a stable periodic orbit [5]. If $O\left(r_{ \pm}^{4}\right)$-terms are neglected, the averaging equation has at most four fixed points:

$$
\left(r_{+}, r_{-}\right)=(0,0), \quad\left(r_{*}, 0\right), \quad\left(0, r_{*}\right), \quad\left(r_{*}, r_{*}\right), \quad r_{*}:=\sqrt{\frac{-\varepsilon \operatorname{Re}\left(p_{1}\right)}{\operatorname{Re}\left(p_{2}\right)}} .
$$

The last three fixed points exist as long as $-\varepsilon \operatorname{Re}\left(p_{1}\right) / \operatorname{Re}\left(p_{2}\right)>0$. The Jacobi matrices of the system at the fixed points are given by

$$
\varepsilon \operatorname{Re}\left(p_{1}\right)\left(\begin{array}{ll}
1 & 0 \\
0 & 1
\end{array}\right), \varepsilon \operatorname{Re}\left(p_{1}\right)\left(\begin{array}{cc}
-2 & 0 \\
0 & 1
\end{array}\right), \varepsilon \operatorname{Re}\left(p_{1}\right)\left(\begin{array}{cc}
1 & 0 \\
0 & -2
\end{array}\right), \varepsilon \operatorname{Re}\left(p_{1}\right)\left(\begin{array}{cc}
-2 & 0 \\
0 & -2
\end{array}\right)
$$

respectively. Because of the assumption (A4) and Lemma 3.3, we have $\operatorname{Re}\left(p_{1}\right)>0$. This shows that when $\varepsilon=K-K_{c}<0$, the point $(0,0)$ is 
stable, and when $\varepsilon=K-K_{c}>0$ and $\operatorname{Re}\left(p_{2}\right)<0$, the fixed point $\left(r_{*}, r_{*}\right)$ exists and is stable. This proves that when $\varepsilon>0$ and $\operatorname{Re}\left(p_{2}\right)<0$, the averaging equation (6.13) has a stable fixed point $\left(r_{+}, r_{-}\right)=\left(r_{*}, r_{*}\right)+O(\varepsilon)$, and the system (6.11) has a family of stable periodic orbits

$$
\left(\alpha_{+}, \alpha_{-}\right)=\left(r_{*} e^{i\left(y_{c} t+O(\varepsilon)\right)+i \beta}+O(\varepsilon), r_{*} e^{-i\left(y_{c}+O(\varepsilon)\right)+i \beta}+O(\varepsilon)\right),
$$

where $\beta \in \mathbb{R}$ is an arbitrary constant induced by the action $\left(\alpha_{+}, \alpha_{-}\right) \mapsto$ $\left(e^{i \beta} \alpha_{+}, e^{i \beta} \alpha_{-}\right)$and it is specified by an initial condition. Since the order parameter is $\eta_{1}=\alpha_{+}+\alpha_{-}+O\left(\alpha^{2}\right)$, we obtain a family of stable solutions

$$
\eta_{1}=2 r_{*} e^{i \beta} \cos \left(y_{c} t+O(\varepsilon)\right)+O(\varepsilon) .
$$

This completes the proof of Theorem 1.3 (i).

\subsection{Center manifold reduction $(h \neq 0)$}

Assume $h \neq 0$. Then, $T_{2}=2 i \omega+h K \mathcal{P}$. We again assume (6.5), and make the following ansatz

$$
\varepsilon \sim O(\alpha), \quad \frac{d \alpha_{ \pm}}{d t}= \pm i y_{c} \alpha_{ \pm}+O\left(\alpha^{2}\right)
$$

Lemma 6.3. Define

$$
\begin{aligned}
Z_{2}= & \frac{K_{c}^{2}}{4} \alpha_{+}^{2} \mu_{+}^{2}+\frac{K_{c}^{2}}{4} \alpha_{-}^{2} \mu_{-}^{2}-\frac{K_{c}^{2}}{4 i y_{c}} \alpha_{+} \alpha_{-}\left(\mu_{+}-\mu_{-}\right) \\
& -\frac{h K_{c}^{3}}{8} \frac{D^{\prime}\left(i y_{c}\right)}{1-h} \alpha_{+}^{2} \mu_{+}-\frac{h K_{c}^{3}}{8} \frac{D^{\prime}\left(-i y_{c}\right)}{1-h} \alpha_{-}^{2} \mu_{-}+O\left(\alpha^{3}\right) .
\end{aligned}
$$

It satisfies the second differential equation of (6.3) up to the order $O\left(\alpha^{3}\right)$.

This is proved in a similar manner to Lemma 6.2. Let us apply the projection $\Pi_{c}$ to the both sides of the first equation of Eq.(6.3).

$$
\frac{K_{c}}{2}\left(\dot{\alpha}_{+} \mu_{+}+\dot{\alpha}_{-} \mu_{-}\right)=T_{c}^{\times} \Pi_{c} Z_{1}+\frac{\varepsilon}{2} \eta_{1} \Pi_{c} P_{0}+\frac{K_{c} h}{2} \eta_{2} \Pi_{c} Z_{-1}+O\left(\alpha^{3}\right) .
$$

Lemma 6.3 with Lemma 6.1 gives

$$
\begin{aligned}
\eta_{2}=\left(Z_{2}, P_{0}\right)= & -\frac{K_{c}^{2}}{4} \alpha_{+}^{2} D^{\prime}\left(i y_{c}\right)-\frac{K_{c}^{2}}{4} \alpha_{-}^{2} D^{\prime}\left(-i y_{c}\right) \\
& -\frac{h K_{c}^{2}}{4} \frac{D^{\prime}\left(i y_{c}\right)}{1-h} \alpha_{+}^{2}-\frac{h K_{c}^{2}}{4} \frac{D^{\prime}\left(-i y_{c}\right)}{1-h} \alpha_{-}^{2}+O\left(\alpha^{3}\right) \\
= & -\frac{K_{c}^{2}}{4} \frac{D^{\prime}\left(i y_{c}\right)}{1-h} \alpha_{+}^{2}-\frac{K_{c}^{2}}{4} \frac{D^{\prime}\left(-i y_{c}\right)}{1-h} \alpha_{-}^{2}+O\left(\alpha^{3}\right) .
\end{aligned}
$$


Lemma 6.4. $\Pi_{c} Z_{-1}$ is given by

$$
\Pi_{c} Z_{-1}=\frac{-4}{K_{c} D^{\prime}\left(i y_{c}\right)} e^{-i \arg \left(\alpha_{+}\right)} \mu_{+}+\frac{-4}{K_{c} D^{\prime}\left(-i y_{c}\right)} e^{-i \arg \left(\alpha_{-}\right)} \mu_{-}+O(\alpha) .
$$

See [1] for the proof.

Substituting these equalities into Eq. 6.17) and comparing the coefficients of $\mu_{+}$and $\mu_{-}$, respectively, in the both sides of the equation, we obtain the dynamics on the center manifold

$$
\left\{\begin{array}{l}
\frac{d \alpha_{+}}{d t}=i y_{c} \alpha_{+}+q_{1} \varepsilon\left(\alpha_{+}+\alpha_{-}\right)+\left(q_{2} \alpha_{+}^{2}+q_{3} \alpha_{-}^{2}\right) e^{-i \arg \left(\alpha_{+}\right)}+O\left(\alpha^{3}\right), \\
\frac{d \alpha_{-}}{d t}=-i y_{c} \alpha_{-}+\bar{q}_{1} \varepsilon\left(\alpha_{+}+\alpha_{-}\right)+\left(q_{2} \alpha_{-}^{2}+\bar{q}_{3} \alpha_{+}^{2}\right) e^{-i \arg \left(\alpha_{-}\right)}+O\left(\alpha^{3}\right),
\end{array}\right.
$$

where $q_{1}, q_{2}$ and $q_{3}$ are complex numbers defined by

$$
q_{1}=\frac{-2}{K_{c}^{2} D^{\prime}\left(i y_{c}\right)}, \quad q_{2}=\frac{h K_{c}}{1-h}, \quad q_{3}=\frac{h K_{c}}{1-h} \frac{\overline{D^{\prime}\left(i y_{c}\right)}}{D^{\prime}\left(i y_{c}\right)} .
$$

( $q_{1}$ is the same number as $p_{1}$ ). Note that $q_{2}$ is a real number. The next purpose is to reduce this system by the same way as the last section. Since the system is invariant under the action $\left(\alpha_{+}, \alpha_{-}\right) \mapsto\left(e^{i \beta} \alpha_{+}, e^{i \beta} \alpha_{-}\right)$for $\beta \in \mathbb{R}$, we can assume without loss of generality that $\arg \left(\alpha_{+}\right)+\arg \left(\alpha_{-}\right)=0$. Hence, we put $\alpha_{ \pm}=r_{ \pm} e^{ \pm i \psi}$ with $r_{ \pm}, \psi \in \mathbb{R}$. Substituting this into the system, we obtain the three dimensional system

$$
\left\{\begin{array}{l}
\dot{\psi}=y_{c}+O(\varepsilon), \\
\dot{r}_{+}=\varepsilon \operatorname{Re}\left(q_{1}\right) r_{+}+\varepsilon \operatorname{Re}\left(q_{1} e^{-2 i \psi}\right) r_{-}+q_{2} r_{+}^{2}+\operatorname{Re}\left(q_{3} e^{-4 i \psi}\right) r_{-}^{2}+O\left(r_{ \pm}^{3}\right), \\
\dot{r}_{-}=\varepsilon \operatorname{Re}\left(q_{1}\right) r_{-}+\varepsilon \operatorname{Re}\left(q_{1} e^{-2 i \psi}\right) r_{+}+q_{2} r_{-}^{2}+\operatorname{Re}\left(q_{3} e^{-4 i \psi}\right) r_{+}^{2}+O\left(r_{ \pm}^{3}\right) .
\end{array}\right.
$$

Now we apply the averaging method. The right hand sides of the equations of $r_{+}$and $r_{-}$are averaged over $\psi$ to obtain the averaging equation

$$
\left\{\begin{array}{l}
\dot{r}_{+}=\varepsilon \operatorname{Re}\left(q_{1}\right) r_{+}+q_{2} r_{+}^{2}+O\left(r_{ \pm}^{3}\right) \\
\dot{r}_{-}=\varepsilon \operatorname{Re}\left(q_{1}\right) r_{-}+q_{2} r_{-}^{2}+O\left(r_{ \pm}^{3}\right)
\end{array}\right.
$$

If $O\left(r_{ \pm}^{3}\right)$-terms are neglected, the averaging equation has at most four fixed points:

$$
\left(r_{+}, r_{-}\right)=(0,0), \quad\left(r_{*}, 0\right), \quad\left(0, r_{*}\right), \quad\left(r_{*}, r_{*}\right), \quad r_{*}:=\frac{-\varepsilon \operatorname{Re}\left(q_{1}\right)}{q_{2}} .
$$


The last three fixed points exist only when $-\varepsilon \operatorname{Re}\left(q_{1}\right) / q_{2}>0$. The Jacobi matrices of the system at the fixed points are given by

$\varepsilon \operatorname{Re}\left(q_{1}\right)\left(\begin{array}{ll}1 & 0 \\ 0 & 1\end{array}\right), \varepsilon \operatorname{Re}\left(q_{1}\right)\left(\begin{array}{cc}-1 & 0 \\ 0 & 1\end{array}\right), \varepsilon \operatorname{Re}\left(q_{1}\right)\left(\begin{array}{cc}1 & 0 \\ 0 & -1\end{array}\right), \varepsilon \operatorname{Re}\left(q_{1}\right)\left(\begin{array}{cc}-1 & 0 \\ 0 & -1\end{array}\right)$,

respectively. Because of the assumption (A4) and Lemma 3.3, we have $\operatorname{Re}\left(q_{1}\right)>0$. This shows that when $\varepsilon=K-K_{c}<0$, the point $(0,0)$ is stable. When $K-K_{c}<0$ and $q_{2}>0$, the fixed points $\left(r_{*}, 0\right),\left(0, r_{*}\right),\left(r_{*}, r_{*}\right)$ exist but they are unstable. When $K-K_{c}>0$ and $q_{2}<0$, the fixed point $\left(r_{*}, r_{*}\right)$ is stable. Since $h<1$ (the assumption (A1)), $q_{2}<0$ is equivalent to $h<0$. This proves that when $K-K_{c}>0$ and $h<0$, the averaging equation (6.20) has a stable fixed point $\left(r_{+}, r_{-}\right)=\left(r_{*}, r_{*}\right)+O\left(\varepsilon^{2}\right)$, and the system (6.18) has a family of stable periodic orbits

$$
\left(\alpha_{+}, \alpha_{-}\right)=\left(r_{*} e^{i\left(y_{c} t+O(\varepsilon)\right)+i \beta}+O\left(\varepsilon^{2}\right), r_{*} e^{-i\left(y_{c}+O(\varepsilon)\right)+i \beta}+O\left(\varepsilon^{2}\right)\right),
$$

where $\beta \in \mathbb{R}$ is an arbitrary constant induced by the action $\left(\alpha_{+}, \alpha_{-}\right) \mapsto$ $\left(e^{i \beta} \alpha_{+}, e^{i \beta} \alpha_{-}\right)$and it is specified by an initial condition. Since the order parameter is $\eta_{1}=\alpha_{+}+\alpha_{-}+O\left(\alpha^{2}\right)$, we obtain a family of stable solutions

$$
\eta_{1}=2 r_{*} e^{i \beta} \cos \left(y_{c} t+O(\varepsilon)\right)+O\left(\varepsilon^{2}\right) .
$$

This completes the proof of Theorem 1.3 (ii).

\section{References}

[1] H.Chiba, I.Nishikawa, Center manifold reduction for a large population of globally coupled phase oscillators, Chaos, 21, 043103 (2011).

[2] H. Chiba, A proof of the Kuramoto conjecture for a bifurcation structure of the infinite-dimensional Kuramoto model, Ergodic Theory Dynam. Systems 35 (2015), no. 3, 762-834.

[3] H. Chiba, A spectral theory of linear operators on rigged Hilbert spaces under analyticity conditions, Adv. in Math. 273, 324-379, (2015).

[4] H. Chiba, A center manifold reduction of the Kuramoto-Daido model with a phase-lag, arXiv:1609.04126).

[5] H. Chiba, Extension and unification of singular perturbation methods for ODEs based on the renormalization group method, SIAM j. on Appl. Dyn.Syst., Vol.8, 1066-1115 (2009).

[6] H. Daido, Onset of cooperative entrainment in limit-cycle oscillators with uniform all-to-all interactions: bifurcation of the order function, Phys. D 91, no. 1-2, 24-66, (1996). 
[7] T. Kato, Perturbation theory for linear operators, Springer-Verlag, Berlin, 1995.

[8] E. A. Martens, E. Barreto, S. H. Strogatz, E. Ott, P. So, T. M. Antonsen, Exact results for the Kuramoto model with a bimodal frequency distribution, Phys. Rev. E (3) 79, 026204 (2009).

[9] E. Ott, T. M. Antonsen, Low dimensional behavior of large systems of globally coupled oscillators, Chaos 18, 037113 (2008).

[10] A. Pikovsky, M. Rosenblum, J. Kurths, Synchronization: A Universal Concept in Nonlinear Sciences, Cambridge University Press, Cambridge, 2001.

[11] K. Yosida, Functional analysis, Springer-Verlag, Berlin, 1995. 